\title{
FOX-NEUWIRTH CELL STRUCTURES AND THE COHOMOLOGY OF SYMMETRIC GROUPS
}

\author{
CHAD GIUSTI AND DEV SINHA
}

\begin{abstract}
We use the Fox-Neuwirth cell structure for one-point compactifications of configuration spaces as the starting point for understanding our recent calculation of the mod-two cohomology of symmetric groups. We then use that calculation to give short proofs of classical results on this cohomology due to Nakaoka and to Madsen.
\end{abstract}

\section{INTRODUCTION}

Group cohomology touches on a range of subjects within algebra and topology. It is thus amenable to study by a similar range of techniques, as we have found in our recent work on the cohomology of symmetric groups. The key organizational tool is the algebraic notion of a Hopf ring. But it is the geometry of bundles and that of the corresponding models for classifying spaces which have guided us, and in particular led to rediscovery of the Hopf ring structure.

We consider cohomology of symmetric groups as giving characteristic classes for finite-sheeted covering maps. Equivalently, we consider the configuration space models for their classifying spaces. There is a strong analogy with characteristic classes for vector bundles and the geometry of Grassmannians. In the vector bundle setting, we embed a bundle over a manifold base space in a trivial bundle and define characteristic classes which are Poincaré dual to the locus where the fiber intersects the standard flag in subspaces with a prescribed set of dimensions. These cohomology classes are pulled back from classes represented by Schubert cells. We may use the isomorphism $O(1)=\mathcal{S}_{2}$, to translate to covering spaces. Embed a two-sheeted covering of a manifold once again in a trivial vector bundle. Then the characteristic classes, which are all powers of the first Stiefel-Whitney class, are Poincaré dual to the locus where the two points in a fiber share their first $n$ coordinates. We will see that in general characteristic classes of finite sheeted covering spaces are defined by loci where the points in the fiber are (nested) partitioned into collections of points which share prescribed coordinates.

A critical component of the the theory of characteristic classes for vector bundles is the Schubert cell structure on Grassmannians. We begin this paper by developing an analogue for configuration spaces, namely the Fox-Neuwirth cell structure on their one-point compacitifications. While this cell structure in the two-dimensional setting dates back fifty years 14 and was developed in all dimensions mod-two by Vassiliev [30, we present what is to our knowledge the first treatment of the differential integrally. This cell structure is "Alexander dual" to one on configuration spaces themselves which has enjoyed a renaissance of interest lately, being studied in a number of contexts, namely by Tamaki [26, 27, related to work on the iterated cobar construction [29, 28, by Ayala and Hepworth [3] in the context of higher category theory and by Blagojević and Ziegler [8] in the context of partitioning convex bodies. After presenting the cell structure, we compute the mod-two cohomology of $B \mathcal{S}_{4}$, giving cochain representatives.

In order to understand arbitrary symmetric groups, we find it essential to consider them all together, much as in homology where their direct sum has an elegant description as a "Q-ring" [7. In cohomology the relevant structure is that of a Hopf ring, due to Strickland and Turner [25. This was the key organizing structure of our work with Salvatore in [15. Our construction of a model for this Hopf ring structure at the level of Fox-Neuwirth cochains is new. After giving a Hopf ring presentation in terms of generators 
and relations, we give a graphical presentation of the resulting monomial basis which we call the skyline diagram basis, recapping the major results from our paper [15] and connecting them with Fox-Neuwirth cochain representatives first identified by Vassiliev 30. We use the skyline diagram presentation to revisit the structure of the cohomology of $S_{\infty}$ as an algebra over the Steenrod algebra, and in particular to see the Dickson algebras as an associated graded.

\section{Contents}

1. Introduction

2. The Fox-Neuwirth cochain complexes

2.1. Fox-Neuwirth cells

2.2. The cochain complexes

3. The cohomology of $B \mathcal{S}_{4}$

3.1. Block symmetry and skyline diagrams

3.2. Geometry and characteristic classes

4. Hopf ring structure and presentation

4.1. Cup product structure

4.2. Hopf ring structure

4.3. Hopf ring presentation

5. The skyline basis

6. The cohomology of $B \mathcal{S}_{\infty}$ as an algebra over the Steenrod algebra

6.1. Nakaoka's theorem revisited

\begin{tabular}{llr} 
6.2. Steenrod structure & 15 \\
\hline
\end{tabular}

6.3. Madsen's theorem revisited $\quad 16$

References

\section{The Fox-Neuwirth COCHAin COMPLEXeS}

In this section we decompose configuration spaces into open cells, analogously to how one decomposes Grassmannians into Schubert cells. Consider $\mathbb{R}^{m}$ with its standard basis, and let Conf ${ }_{n}\left(\mathbb{R}^{m}\right)$ denote the space of configurations of $n$ distinct, labelled points in $\mathbb{R}^{m}$ topologized as a subspace of $\mathbb{R}^{m n}$. The configuration space $\operatorname{Conf}_{n}\left(\mathbb{R}^{m}\right)$ admits a free, transitive action of $\mathcal{S}_{n}$, the symmetric group on $n$ letters, by permutation of the labels. We denote by $\overline{\operatorname{Conf}}_{n}\left(\mathbb{R}^{m}\right)$ the quotient $\operatorname{Conf}_{n}\left(\mathbb{R}^{m}\right) / \mathcal{S}_{n}$, which is the space of unlabeled configurations of $n$ points in $\mathbb{R}^{m}$.

The standard embeddings $\mathbb{R}^{m} \hookrightarrow \mathbb{R}^{m+1}$ give rise to a canonical directed system of configuration spaces $\operatorname{Conf}_{n}\left(\mathbb{R}^{2}\right) \hookrightarrow \operatorname{Conf}_{n}\left(\mathbb{R}^{3}\right) \hookrightarrow \cdots$ whose maps are all equivariant with respect to the $\mathcal{S}_{n}$ action. We denote the limit by $\operatorname{Conf}_{n}\left(\mathbb{R}^{\infty}\right)$. A map from a sphere of dimension less than $m-1$ to Conf ${ }_{n}\left(\mathbb{R}^{m}\right)$ can be first nullhomotoped in $\mathbb{R}^{m n}$, but then by general position be null-homotoped in $\operatorname{Conf}_{n}\left(\mathbb{R}^{m}\right)$ itself. Thus Conf ${ }_{n}\left(\mathbb{R}^{m}\right)$ is $m-2$ connected, so $\operatorname{Conf}_{n}\left(\mathbb{R}^{\infty}\right)$ is weakly contractible. Moreover $\operatorname{Conf}_{n}\left(\mathbb{R}^{\infty}\right)$ inherits a free action of $\mathcal{S}_{n}$, so it is a model for $E \mathcal{S}_{n}$. Thus $\overline{\operatorname{Conf}}_{n}\left(\mathbb{R}^{\infty}\right) \simeq B \mathcal{S}_{n}$. Indeed, $B \mathcal{S}_{n}$ classifies finite-sheeted covering spaces, and by embedding such a bundle over some paracompact base in a trivial Euclidean bundle we can define the classifying map to $\overline{\operatorname{Conf}}_{n}\left(\mathbb{R}^{\infty}\right)$.

2.1. Fox-Neuwirth cells. We now describe "open" cellular decompositions of Conf ${ }_{n}\left(\mathbb{R}^{m}\right)$ and $\overline{\operatorname{Conf}}_{n}\left(\mathbb{R}^{m}\right)$ which then define CW-structures on their one-point compactifications. These cellular decompositions are due to Fox and Neuwirth [14] when $m=2$, were considered by Vassiliev in higher dimensions [30], and have also been considered in the context of $E_{m}$-operads by Berger [5, 6]. They are in some sense Alexander dual to the Milgram decompositions of configuration spaces [20] (and more generally the Salvetti complex 


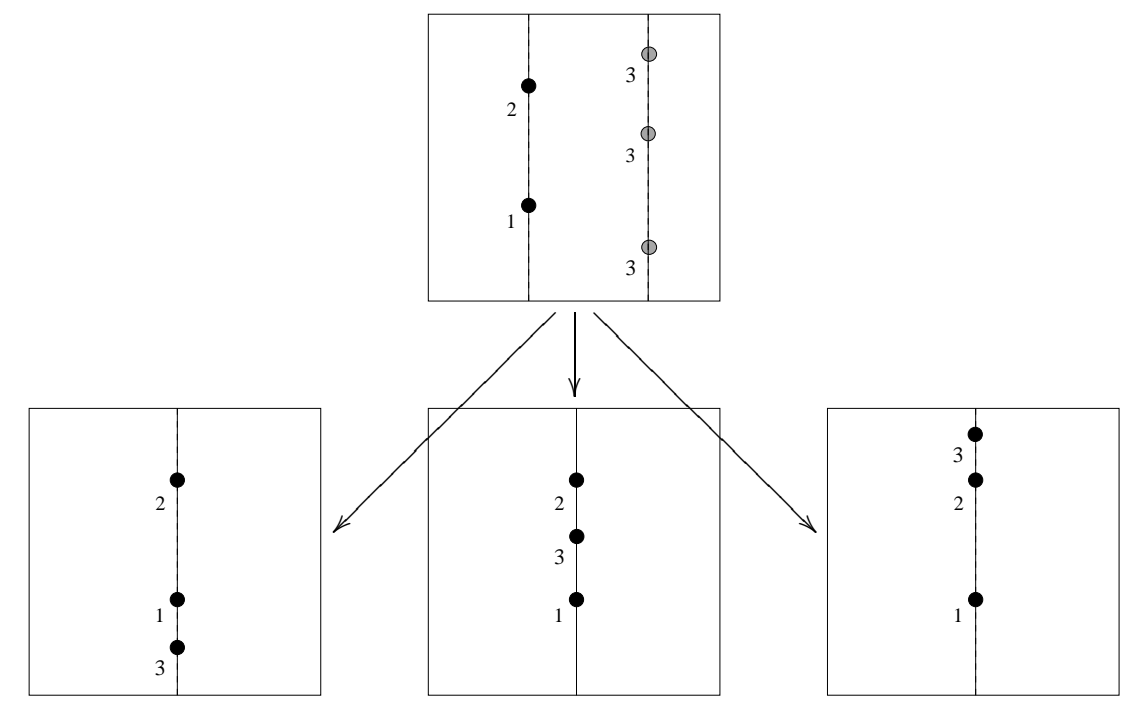

FIgURE 1. The degeneration of the second inequality in the cell $1<_{1} 2<_{0} 3$ to the bounding cells $3<_{1}<1<_{1} 2,1<_{1} 3<_{1} 2$ and $1<_{1} 2<_{1} 3$ respectively .

[23]) and the realizations of $\theta$-categories of Joyal [18. In the limit these cellular decompositions give rise to a cochain complex to compute the cohomology of symmetric groups - see Theorem 2.9.

The cell structure is based on the dictionary ordering of points in $\mathbb{R}^{m}$ using standard coordinates, which we denote by $<$. This ordering gives rise to an ordering of points in a configuration.

Definition 2.1. A depth-ordering is a series of labeled inequalities $i_{1}<_{a_{1}} i_{2}<_{a_{2}} i_{3}<_{a_{3}} \cdots<_{a_{n-1}} i_{n}$, where the $a_{i}$ are non-negative integers and the set of $\left\{i_{k}\right\}$ is exactly $\{1, \ldots, n\}$.

For any depth-ordering $\Gamma$ define $\operatorname{Conf}_{\Gamma}\left(\mathbb{R}^{m}\right)$ to be the collection of all configurations $\left(x_{1}, x_{2}, \ldots, x_{n}\right) \in$ $\operatorname{Conf}_{n}\left(\mathbb{R}^{m}\right)$ such that for any $p, x_{i_{p}}<x_{i_{p+1}}$ and their first $a_{p}$ coordinates are equal while their $\left(a_{p}+1\right)$ st coordinate must differ.

The subspace $\operatorname{Conf}_{\Gamma}\left(\mathbb{R}^{m}\right)$ is empty if any $a_{i}$ is greater than or equal to $m$.

Theorem 2.2 (after Fox-Neuwirth). For any $\Gamma$ the subspace Conf $f_{\Gamma}\left(\mathbb{R}^{m}\right)$ is homeomorphic to a Euclidean ball of dimension $m n-\sum a_{i}$. The images of the $\operatorname{Conf}_{\Gamma}\left(\mathbb{R}^{m}\right)$ are the interiors of cells in an equivariant $C W$ structure on the one-point compactification $\operatorname{Conf} f_{n}\left(\mathbb{R}^{m}\right)^{+}$.

Because this cell structure is equivariant, it descends to one for $\overline{\operatorname{Conf}}_{n}\left(\mathbb{R}^{m}\right)^{+}$, where the cells are indexed only by the $a_{i}$. But to understand the boundary structure, we need to work with ordered configurations. While these cells are very easy to name, their boundary structure is intricate. Consider the cell $\operatorname{Conf}_{\Gamma}\left(\mathbb{R}^{m}\right)$ with $\Gamma=1<_{1} 2<_{0} 3$. It is immediate to see the cell labeled by $1<_{2} 2<_{0} 3$ on the boundary of $\Gamma$, as the inequality between the second coordinates of $x_{1}$ and $x_{2}$ degenerates to an equality. But the cell labeled by $2<_{2} 1<_{0} 3$ is also on the boundary, since the third coordinates of $x_{1}$ and $x_{2}$ are unrestricted as the second coordinates become equal. There are even more possibilities when the inequality between the first coordinates of $x_{2}$ (and thus $x_{1}$ as well) and $x_{3}$ degenerates to an equality. The first inequality, based on the second coordiates of $x_{1}$ and $x_{2}$, will still hold. But the second coordinate of $x_{3}$ has been unrestricted by $\Gamma$, so the cells labeled by $1<_{1} 2<_{1} 3,1<_{1} 3<_{1} 2$ and $3<_{1} 1<_{1} 2$ all occur on the boundary of $\Gamma$. See Figure 1. The combinatorics of shuffles will play a central role in the boundary structure in general. 
We introduce a data structure equivalent to depth orderings in terms of trees in order to more easily describe the boundary combinatorics. These trees were also used by Vassiliev [30] to label this cell structure, and have been of interest in higher category theory (see for example the Chapter 7 of the expository book 11]). Recall that in a rooted tree, one vertex is under another if it is in the unique path from the root to that vertex.

Definition 2.3. For a depth-ordering $\Gamma=i_{1}<_{a_{1}} i_{2}<\cdots<_{a_{n-1}} i_{n}$ define a planar level tree $\tau_{\Gamma}$ up to isotopy as follows.

- There is a root vertex at the origin, and all others vertices have positive integer-valued heights (that is, $y$-coordinates).

- There are $n$ leaves which are of height $m$ labelled $i_{1}, i_{2}, \cdots i_{n}$ in order from left to right (that is, in order of $x$-coordinates).

- Under each leaf there is a unique edge from a vertex of height $i$ to one of height $i+1$ for each $0 \leq i<m$. The leaves labelled $i_{p}$ and $i_{p+1}$ share the edges under them up to height $a_{p}$, but not above this height.

For example, the depth ordering $\Gamma=3<_{2} 5<_{0} 1<_{3} 2<_{0} 4<_{1} 6$ is represented by the following tree.

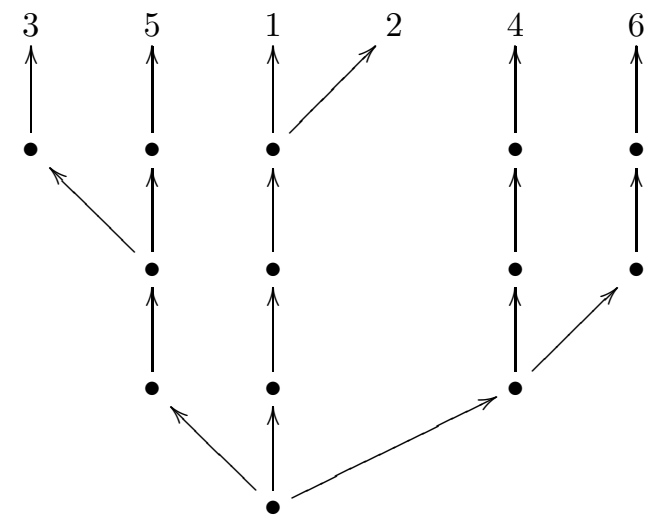

The tree $\tau_{\Gamma}$ uniquely determines $\Gamma$, so we use the two structures interchangeably. Recall as well that the planar isotopy class of a level tree is equivalent to an abstract level tree with an ordering (say from left to right) of the edges incident to each vertex.

Definition 2.4. Let $\tau$ be a planar level tree. If $e$ and $f$ are two edges which are incident to the internal vertex $v$, consecutive in the incident ordering with $e$ before $f$, then the edge quotient $\tau_{/(e=f)}$ is the planar level tree described as an abstract level tree with edge orderings as follows.

- Its edge set is obtained from that of $\tau$ by identifying $e$ and $f$. We call the resulting special edge the quotient edge.

- Its vertex set is obtained from that of $\tau$ by identifying the terminal vertices of $e$ and $f$. We call the resulting vertex the quotient vertex.

- The edges with this terminal vertex as initial are ordered consistently with their previous ordering, with those incident to $e$ before those incident before $f$.

- All other incidence relations and edge orderings are transported by the bijection away from $e$ and $f$ with the edges and vertices of $\tau$.

Definition 2.5. A vertex permutation at $v$ of a planar level tree $\tau$ is a tree which differs from $\tau$ only by changing the edge ordering of $v$. We denote such by $\sigma_{v} \tau$, where $\sigma_{v}$ is the permutation of edges at $v$.

When $\sigma_{v}$ is a shuffle, we call the resulting tree a vertex shuffle of $\tau$. When the tree in question is an edge quotient $\tau_{/(e=f)}$, by convention we shuffle at the quotient vertex $v$ using the initial partition into edges which were incident to $e$ in $\tau$ followed by edges which were incident to $f$. 
We can now describe the boundary structure of Fox-Neuwirth cells.

Proposition 2.6. The cell Conf $\Gamma_{\Gamma^{\prime}}\left(\mathbb{R}^{m}\right)$ is in the boundary of Conf $\Gamma_{\Gamma}\left(\mathbb{R}^{m}\right)$ if and only if $\tau_{\Gamma^{\prime}}$ is isomorphic to $\sigma_{v} \tau_{\Gamma /(e=f)}$ for some consecutive edges e and $f$ and some shuffle $\sigma_{v}$ at the quotient vertex.

For example, let $m=3$ and $\Gamma=1<_{1} 2<_{0} 3$. Then $\Gamma$ is represented by the following tree.

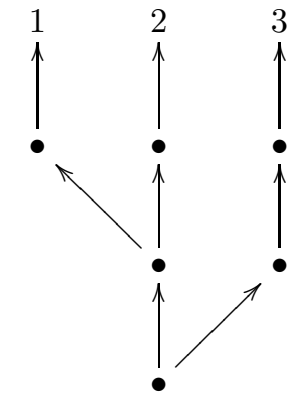

$\operatorname{Conf}_{\Gamma}\left(\mathbb{R}^{3}\right)$ has as its boundary precisely cells $\operatorname{Conf}_{\Gamma^{\prime}}\left(\mathbb{R}^{3}\right)$ with $\Gamma^{\prime}$ represented by each of the following.

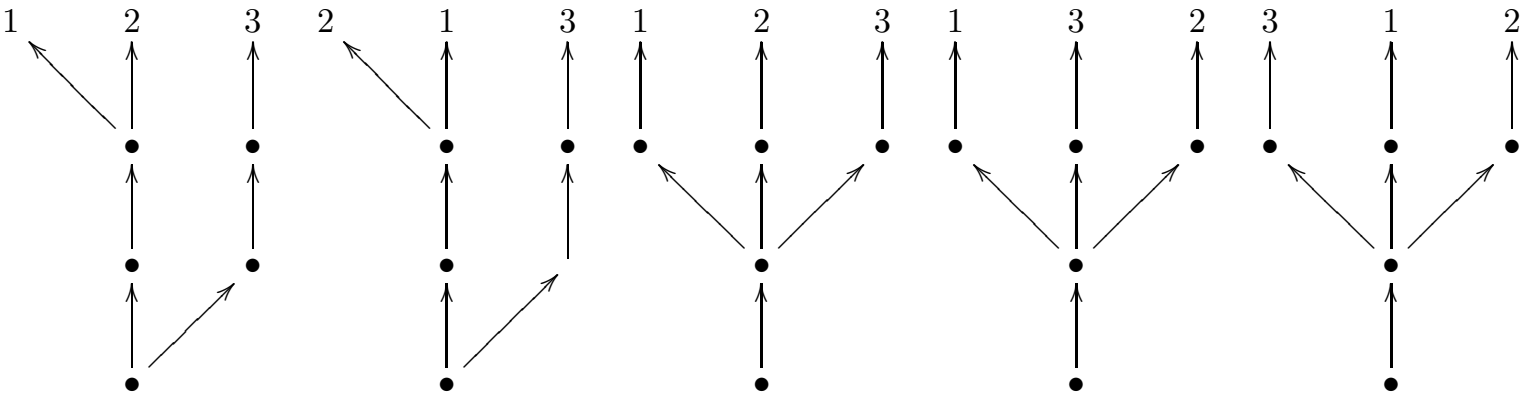

If we had instead considered $\operatorname{Conf}_{\Gamma}\left(\mathbb{R}^{2}\right)$, only the three cells corresponding to the last three in the above list would appear in the boundary since the other potential cells are empty.

There is one edge quotient for each consecutive pair of leaves in the tree $\tau_{\Gamma}$, or equivalently for each inequality in $\Gamma$. For each edge quotient, the number of vertex shuffles varies and is always at least two.

2.2. The cochain complexes. We give what is to our knowledge the first explicit formula for the differential in the resulting integral cochain complexes. In light of Proposition 2.6 it remains to determine signs. Fox-Neuwrith cells are simple to endow with coordinates and thus orientations. Take the standard coordinates of $\operatorname{Conf}_{n}\left(\mathbb{R}^{m}\right)$ as a subspace of $\mathbb{R}^{m n}$, namely $\left(x_{1}\right)_{1},\left(x_{1}\right)_{2}, \ldots,\left(x_{1}\right)_{m},\left(x_{2}\right)_{1}, \ldots$, and omit any coordinate which from the definition of $\operatorname{Conf}_{\Gamma}\left(\mathbb{R}^{m}\right)$ must be equal to one which appears earlier in the list. Straightforward analysis suffices to understand the difference between the orientation of a cell and that induced by a cell which bounds it, leading to the following.

Definition 2.7. The sign of the quotient vertex of the $p$ th edge quotient of $\Gamma=i_{1}<a_{1} i_{2}<\cdots<a_{n-1} i_{n}$, denoted $\operatorname{sgn}_{m}(p), p \in\{1, \ldots, n-1\}$ is defined $\operatorname{ass}_{m}(p, \Gamma)=(-1)^{\kappa}$, where

$$
\kappa=p+\sum_{k=1}^{p-1}\left(m-\min \left\{a_{k}, a_{p}+1\right\}\right)+\left(m-\left(a_{p}+1\right)\right)+\sum_{k=p+1}^{n-1}\left(m-\min \left\{a_{k}, a_{p}\right\}\right) .
$$

If we consider only configurations in $\mathbb{R}^{m}$ with $m$ even, we can delete $m$ from the definition of $\kappa$. We now only concern ourselves with the limit as $m$ goes to infinity. 
Definition 2.8. Define the cochain complex ${\widetilde{\mathrm{FN}_{n}}}^{*}$ as follows. As a free abelian group it is generated by depth-orderings, with $[\Gamma]$ in degree $\sum a_{p}$ when $\Gamma=i_{1}<_{a_{1}} i_{2}<\cdots<_{a_{n-1}} i_{n}$. The differential is

$$
d[\Gamma]=\sum_{p} \operatorname{sgn}_{0}(p, \Gamma) a_{p}[\Gamma], \text { where } a_{p}[\Gamma]=\sum_{\sigma_{v}}\left[\Gamma^{\prime}\right] .
$$

The first sum is over inequalities in $\Gamma$, indexed by $p$, which in turn determine an adjacent edge pair $e, f$ in $\tau_{\Gamma}$. For each such edge pair we sum over all possible vertex shuffles of the quotient tree at the quotient vertex, taking the depth-ordering $\Gamma^{\prime}$ associated to the resulting tree $\sigma_{v} \tau_{\Gamma /(e=f)}$.

Theorem 2.9. ${\widetilde{\mathrm{FN}_{n}}}^{*}$ is a cochain model for $E \mathcal{S}_{n}$, so its quotient by $\mathcal{S}_{n}$ which we call $\mathrm{FN}_{n}{ }^{*}$ has cohomology isomorphic to that of the nth symmetric group.

In algebraic terms, ${\widetilde{\mathrm{FN}_{n}}}^{*}$ is a free $\mathbb{Z}\left[\mathcal{S}_{n}\right]$ resolution of the trivial module. It is a challenge to even show algebraically that ${\widetilde{\mathrm{FN}_{n}}}^{*}$ is a cochain complex, much less that it is acyclic.

Sketch of proof. By Theorem 2.2 , the $[\Gamma]$ naturally span the corresponding cellular chain complex for the one-point compactification $\operatorname{Conf}_{n}\left(\mathbb{R}^{m}\right)^{+}$, with $[\Gamma]$ in $C_{m n-\sum a_{i}}^{C W}\left(\operatorname{Conf}_{n}\left(\mathbb{R}^{m}\right)^{+}\right)$. By Alexander and SpanierWhitead duality for manifolds as explained by Atiyah [2, the resulting homology group is isomorphic to cohomology of $\operatorname{Conf}_{n}\left(\mathbb{R}^{m}\right)$ in degree $k=\sum a_{i}$. These cochain groups are isomorphic through the maps induced by the directed system in degrees $k$ less than $m$, so the inverse limit is just $\widetilde{\mathrm{FN}}_{n}$.

The main work in proof is then to show that the boundary homomorphisms have the indicated signs.

We now consider more closely the quotient complex $\mathrm{FN}_{n}{ }^{*}$ whose cohomology is that of $B \mathcal{S}_{n}$. The equivalence class of some $\Gamma=i_{1}<_{a_{1}}<i_{2}<\cdots<_{a_{n-1}} i_{n}$ modulo $\mathcal{S}_{n}$, which acts by permuting the $i_{k}$, is given by the subscripts of the inequalities which we now denote $\mathbf{a}=\left[a_{1}, \ldots, a_{n-1}\right]$. These correspond to trees $\tau_{\mathbf{a}}$ as described above but with leaves now unlabelled. Thus $\mathrm{FN}_{n}{ }^{*}$ is significantly smaller than the bar complex, having rank in degree $k$ of the number of partitions of $k$ into $n-1$ non-negative integers. We express the differential of $\mathrm{FN}_{n}{ }^{*}$ in terms of $\mathbf{a}$.

Definition 2.10. An $\ell$-block of $\mathbf{a}$ is a maximal (possibly empty) sub-sequence of consecutive $a_{i}$ greater than $\ell$ in $\mathbf{a}$. Denote the ordered collection of all $\ell$-blocks of $\mathbf{a}$ by $B_{\ell}(\mathbf{a})$.

For example, $B_{0}([0,2,6,0,1])=(\emptyset,[2,6], \emptyset,[1])$ and its $B_{1}$ is $(\emptyset,[2,6], \emptyset, \emptyset)$. These collections $B_{\ell}(\mathbf{a})$ correspond to the forests of rooted trees obtained by deleting from $\tau_{\mathbf{a}}$ all vertices of height lower than $\ell$ and their incident edges.

Definition 2.11. Let $\mathbf{a} \in \mathrm{FN}_{n}{ }^{k}$. Denote by $\mathbf{a}[i]$ the element $\left[a_{1}, a_{2}, \ldots, a_{i-1}, a_{i}+1, a_{i+1}, \ldots, a_{n-1}\right] \in$ $\mathrm{FN}_{n}{ }^{k+1}$.

Let $a_{i} \in \mathbf{a}$ and denote by $\mathbf{A}_{i}$ the $a_{i}$-block of $\mathbf{a}[i]$ containing $a_{i}$. Such a block $\mathbf{A}_{i}$ corresponds to a rooted subtree $\tau_{\mathbf{A}_{i}}$ of $\tau_{\mathbf{a}[i]}$. Let $N=\# B_{a_{i}+1}\left(\mathbf{A}_{i}\right)$, the number of trees in the forest obtained by removing the root from $\tau_{\mathbf{A}_{i}}$ along with its incident edges, and let $k$ to be the number of trees in this forest whose roots were incident to the edge $e$ in the tree $\tau_{\mathbf{a}}$ before the edge quotient $e=f$ which produced $\tau_{\mathbf{a}[i]}$. Define $\operatorname{Sh}(\mathbf{a}, i)$ to be the set of $(k, N-k)$-shuffles.

$\operatorname{Sh}(\mathbf{a}, i)$ acts on $\mathbf{a}[i]$ by shuffling the elements of $B_{a_{i}+1}\left(\mathbf{A}_{\mathbf{i}}\right)$. Equivalently, it acts via vertex shuffles on the tree $\tau_{\mathbf{a}[i]}$.

Proposition 2.12. The differential in $\mathrm{FN}_{n}{ }^{*}$ is given by

$$
\delta(\mathbf{a})=\sum_{i=1}^{n-1}(-1)^{i+\alpha(i)} \delta_{i}(\mathbf{a}) \text { where } \delta_{i}(\mathbf{a})=\sum_{\sigma \in S h(\mathbf{a}, i)}(-1)^{\kappa(\sigma, \mathbf{a})} \sigma \cdot \mathbf{a}[i] .
$$


If we define $h(\mathbf{v})$ to be the height in the tree $\tau_{\mathbf{a}}$ of the vertex $\mathbf{v}$ and $\#(\sigma, h)$ to be the total number of transpositions of vertices at height $h$ in $\tau_{\mathbf{A}_{i}}$ which occur when $\sigma$ acts on the tree, then we have

$$
\alpha(i)=\sum_{j=1}^{i-1} \min \left\{a_{j}, a_{i}+1\right\}+a_{i}+1+\sum_{j=i+1}^{n-1} \min \left\{a_{j}, a_{i}\right\}, \text { and } \kappa(\sigma, \mathbf{a})=\sum_{\mathbf{v}}(\#(\sigma, h)-\#(\sigma, h-1)) \cdot h(\mathbf{v}),
$$

where the second sum is indexed over vertices $\mathbf{v}$ in the subtree $\tau_{\mathbf{A}_{i}}$.

The signs and the differential as a whole do not seem to simplify, as evidenced by the following examples of computations, which spite of the superficial similarity in cell names have substantially different boundaries.

$$
\begin{aligned}
& \delta([2,0,1,2])=-3[2,0,2,2]-1[2,1,1,2] \\
& \delta([1,0,2,2])=-1[1,1,2,2]+1[1,2,2,1]+2[2,0,2,2]-1[2,2,1,1] \\
& \delta([0,2,1,2])=-6[0,2,2,2]-1[1,2,1,2]+1[2,1,1,2]-1[2,1,2,1] \\
& \delta([0,1,2,2])=-4[0,2,2,2]-1[1,2,2,1] .
\end{aligned}
$$

The first author has coded a program in Java to compute these differentials. It is appended at the end of the .tex file which was uploaded for the arXiv preprint of this paper, and is also available on his web site.

\section{The Cohomology of $B \mathcal{S}_{4}$}

In this section we establish the following.

Theorem 3.1. There is an additive basis for $H^{*}\left(B \mathcal{S}_{4} ; \mathbb{F}_{2}\right)$ with representatives given by elements of $\mathrm{FN}_{4}{ }^{*}$ of the following forms

- $[a, 0,0]+[0, a, 0]+[0,0, a]$, with $a>0$

- $[a, 0, b]+[b, 0, a]$, with $0<a<b$

- $[b, a, b]$, with $0 \leq a \leq b$.

These Fox-Neuwirth representative cocycles along with the general representatives we find below were also found by Vassiliev [30, though he did not use them to determine multiplicative structure as we do and only sketched the proof that these cocycles form a basis. Because mod-two homology of symmetric groups is well-understood [22, 12, we based our calculations in 15] on knowledge of homology, as did Vassiliev. We take the opportunity to give a complete proof of this result for $B \mathcal{S}_{4}$ to show that a self-contained treatment of cohomology is feasible.

Throughout this section, we label the entries of a basis element of $\mathrm{FN}_{4}{ }^{*}$ by $a, b, c$ with $a \leq b \leq c$.

Proof. That the cochains listed are cocycles is a straightforward calculation using the formula for the differential $\delta$ in the Fox-Neuwirth cochain complex given in Proposition 2.12.

Cocycles which are "disjoint from" (that is, project to zero in) the subspaces on which our generating cocycles are naturally defined are null-homologous, as we will establish through the construction of a chain homotopy operator. Consider the submodule $S$ of $\mathrm{FN}_{4}{ }^{*}$ spanned by cochains whose entries are all positive, and not of the form $[b, a, b],[a, a, b]$ or $[b, a, a]$ with $a \leq b$. We define a chain operator $P: S \rightarrow \mathrm{FN}_{4}{ }^{*}$ of degree -1 which lowers the smallest entry by one or maps to zero, depending on the order of the blocks with respect to the minimum entry. Explicitly, we have the following, where $0<a<b<c$.

- $[a, b, c] \stackrel{P}{\mapsto}[a-1, b, c]$, and $[a, c, b] \stackrel{P}{\mapsto}[a-1, c, b]$, and $[a, b, b] \stackrel{P}{\mapsto}[a-1, b, b]$

- $[b, c, a],[c, b, a]$ and $[b, b, a]$ all map to zero.

- $[b, a, c] \stackrel{P}{\mapsto}[b, a-1, c]$, 
- $[c, a, b]$ maps to zero.

Let $\chi$ be the automorphism of $S$ which exchanges $a$-blocks, which is a chain map. That is $\chi[a, b, c]=$ $[b, c, a]$, while $\chi[b, a, c]=[c, a, b]$, and so forth. We claim that on $S$ the map $P$ is a chain null-homotopy of $\chi$. For example if $b>a+1$ and $c>b+1$ then

$P \delta[a, b, c]=P([a+1, b, c]+[b, c, a+1]+[a, b+1, c]+[a, c, b+1])=[a, b, c]+0+[a-1, b+1, c]+[a-1, c, b+1]$,

while

$$
\delta P[a, b, c]=\delta[a-1, b, c]=[a, b, c]+[b, c, a]+[a-1, b+1, c]+[a-1, c, b+1],
$$

so their sum is $[b, c, a]$. Removing the restrictions $b>a+1$ and $c>b+1$ and treating other cases all follows from similar direct computation.

Thus for any cocycle $\gamma$ in $S$,

$$
\delta(P \chi(\gamma))=P(\delta \chi(\gamma))+\chi(\chi(\gamma))=\gamma
$$

so any cocycle in $S$ is trivial in cohomology.

We now show that a cycle is always homologous to one whose image in subspaces complementary to $S$ is given by the cycles we have named, which seems to require ad-hoc analysis.

Consider chains whose first entry is zero, but whose second and third are not, which we call $S_{f 0}$. When we compose the differential with projection onto $S_{f 0}$, we obtain a complex which we claim is acyclic, as can be seen using a "decrease the smallest nonzero entry" nullhomtopy as above. Thus any chain which projects to $S_{f 0}$ non-trivially is homologous to one which projects to it trivially. Similarly, we can rule out cycles involving chains whose last entry is zero but first two are not, namely $S_{l 0}$.

For chains whose middle entry alone is zero $S_{m 0}$, we have $\delta[a, 0, b]=[a, 1, b]+[b, 1, a]$. Cochains with a middle entry of one do not otherwise appear in the image of $\delta$, so the projection of a cycle onto $S_{m 0}$ must itself be a cycle, which direct calculation shows must be of the form $[a, 0, b]+[b, 0, a]$.

Similarly, if we consider chains with only one-nonzero entry and two zeros, which we call $S_{00}$, the boundary involves chains with a single zero and a single one. Only chains with two zeros can have such boundaries, so the projection of a cycle onto $S_{00}$ must itself be a cycle, which direct calculation shows must be of the form $[a, 0,0]+[0, a, 0]+[0,0, a]$.

Finally, consider $S_{2 a}$, which is the span of $[a, a, b],[a, b, a]$, and $[a, a, b]$. Assume at first that $a+1<b$, in which case the image of $[a, a, b]$ under $\delta$ is $[a, a+1, b]+[a, b, a+1]$. The only other cochain for which either of these appears non-trivially in its coboundary is $[a, b, a]$, whose coboundary consists of those along with $[b, a+1, a]$ and $[a+1, b, a]$. These latter two terms are the coboundary of $[b, a, a]$, so the only cycle in which these occur nontrivially is $[a, a, b]+[a, b, a]+[a, a, b]$. But this is trivial in cohomology, equal to $\delta([a, a-1, b])$. Thus any cycle is homologous to one which projects trivially onto $S_{2 a}$. The analysis with $a+1=b$ is similar.

Because $\mathrm{FN}_{4}{ }^{*}$ is spanned by $S, S_{f 0}, S_{l 0}, S_{m 0}, S_{00}, S_{2 a}$, and the cycles listed in the statement of the theorem, the result follows.

3.1. Block symmetry and skyline diagrams. The cochain representatives for the cohomology of $B \mathcal{S}_{4}$ all exhibit some symmetry. Define a block permutation to be a permutation of the entries of a FoxNeuwirth basis cochain which does not change its (unordered) set of $i$-blocks for any $i$. The Fox-Neuwirth cochains which comprise each of the cocyles listed in Theorem 3.1 are invariant under block-permutations. In particular, if we let $\operatorname{Symm}(\mathbf{a})$ denote the sum of all distinct block-permutations of a, then the first two sets of generators are $\operatorname{Symm}([a, 0, b])$ for $0 \leq a<b$ and the third is $\operatorname{Symm}([b, a, b])$, for $0 \leq a \leq b$. Symmetric representatives exist for the cohomology $B \mathcal{S}_{n}$ more generally, as we will see below. It might be enlightening to have a more direct proof of this block-symmetry property than through simply observing it holds after full computation. 


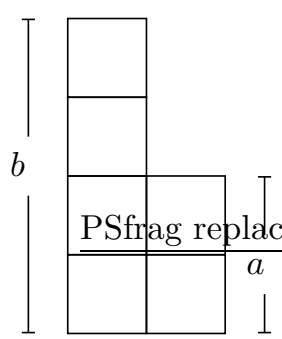

(a) $\operatorname{Symm}([a, 0, b]), 0 \leq a<b$

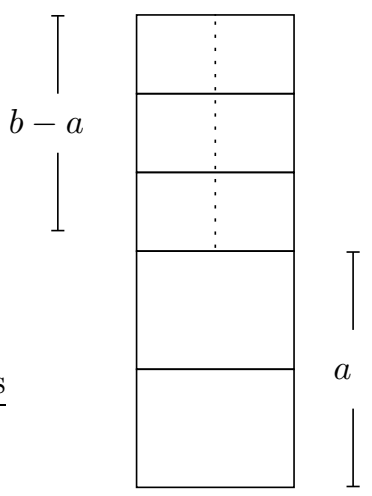

(b) $[b, a, b], 0 \leq a \leq b$

Figure 2. Skyline diagrams for the basis of $H^{*}\left(B \mathcal{S}_{4} ; \mathbb{F}_{2}\right)$ described in Theorem 3.1

The symmetry of these cochains allows the essential data defining them to be given in a graphical representation which we call skyline diagrams. The basis elements described in Theorem 3.1 correspond to skyline diagrams in Figure 2. These will be treated fully in Section 5 .

3.2. Geometry and characteristic classes. We can understand our cocycles geometrically through intersection theory, which is one of the standard ways to understand duality. If $M$ is a manifold without boundary and $M^{+}$is its one-point compactification, then at the cochain level Alexander-Spanier-Whitehead duality identifies a cellular codimension- $d$ cell $C$ in $M^{+}$through a zig-zag of maps with a cochain on $M$ whose value on chains $\sigma: \Delta^{d} \rightarrow M$ which are transversal to the interior of $C$ is the mod-two count of $\sigma^{-1}(C)$. In our setting, these counts are of configurations whose points share prescribed coordinates.

For example, in $H_{4}\left(B \mathcal{S}_{4}\right)$ there is the fundamental class of a submanifold $\mathbb{R} P^{2} \times \mathbb{R} P^{2}$ of $\operatorname{Conf}_{4}\left(\mathbb{R}^{\infty}\right)$. This submanifold parameterizes configurations of four points two of which are on the unit sphere say centered at the origin and two of which are on a unit sphere chosen to be disjoint from that one. The cocycle $[2,0,2]$ evaluates non-trivially on this homology class, as can be seen by a count of configurations in this submanifold and in the cell, as we picture in Figure 3,

The corresponding characteristic class can be evaluated on a four-sheeted covering space by embedding it in a trivial Euclidean bundle and taking the Thom class of the locus where the fibers of the cover can be partitioned into two groups of two, each of which share their first two coordinates. For the cocyle $[2,1,2]$, the corresponding characteristic class would be the Thom class of the locus where all four points in the fiber share their first coordinate, and they can be partitioned into two groups of two which share an additional coordinate.

\section{Hopf Ring STRUCTURE AND PRESENTATION}

While cup product structure typically clarifies the calculation of cohomology, the cohomology rings of symmetric groups have been notoriously difficult to understand. Feshbach's description [13] is complicated, with recursively presented relations. We have found that the situation is clarified once one uses a second product structure on cohomology, along with a coproduct (and antipode which is trivial) in order to obtain what is known as a Hopf ring. This structure was first discovered by Strickland and Turner [25].

\subsection{Cup product structure.}




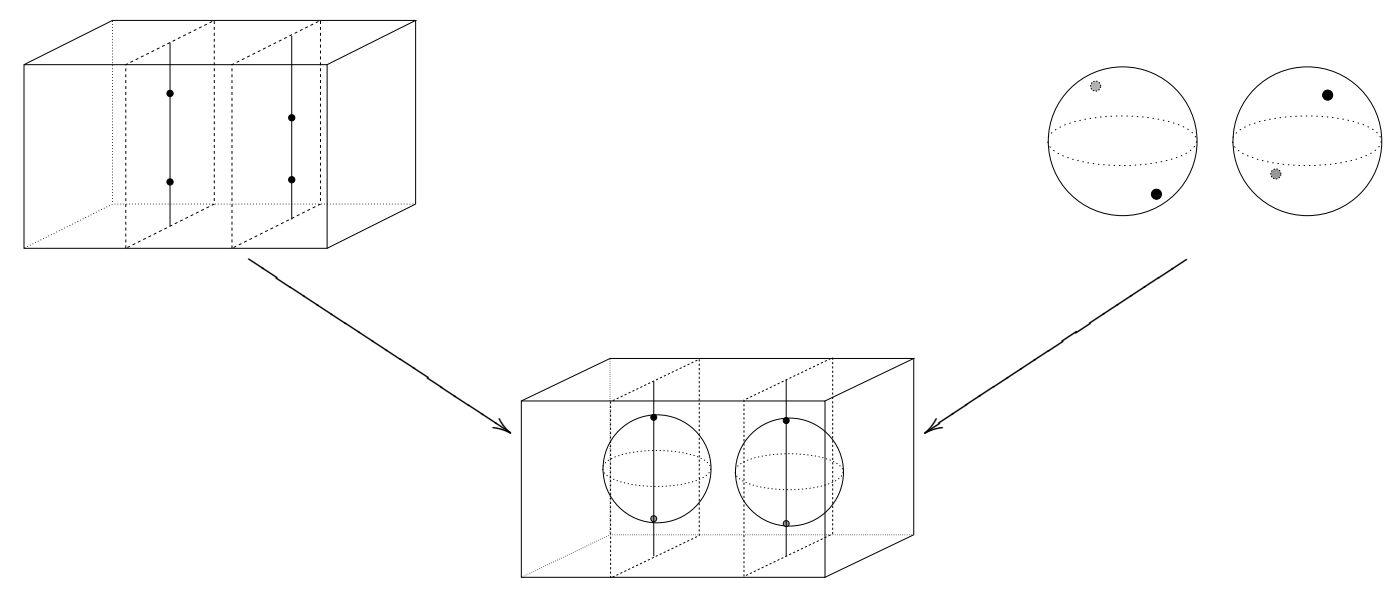

Figure 3 . The cocycle $[2,0,2] \in H^{4}\left(B \mathcal{S}_{4}\right)$ pairs non-trivially with the fundamental class of $\mathbb{R} P^{2} \times \mathbb{R} P^{2}$ considered as a submanifold of $\operatorname{Conf}_{4}\left(\mathbb{R}^{\infty}\right)$ as indicated.

Definition 4.1. Let $\mathbf{a}=\left[a_{1}, \ldots, a_{n-1}\right]$ and $\mathbf{b}=\left[b_{1}, \ldots, b_{n-1}\right]$ be basis elements in the mod-two FoxNeuwirth cochain complex. Define their intersection product $\mathbf{a} \cdot \mathbf{b}$ to be

$$
\left[a_{1}, \ldots, a_{n-1}\right] \cdot\left[b_{1}, \ldots, b_{n-1}\right]=\left[a_{1}+b_{1}, \ldots, a_{n-1}+b_{n-1}\right],
$$

Integrally, we would also have the usual sign accounting for the possible difference in orientations.

Lemma 4.2. The intersection product makes $\mathrm{FN}^{*}$ a (commutative) differential graded algebra.

Proposition 4.3. Through the isomorphism of Theorem [2.9, the product on cohomology induced by the intersection product on $\mathrm{FN}_{n}^{*}$ agrees with the cup product on cohomology of $B \mathcal{S}_{n}$.

We call this the intersection product in light of the fact mentioned above that the cohomology classes which arise from the Fox-Neuwirth cell structure on $\overline{\operatorname{Conf}}_{n}\left(\mathbb{R}^{D}\right)^{+}$are Thom classes of the union of corresponding cells in $\overline{\operatorname{Conf}}_{n}\left(\mathbb{R}^{D}\right)$. These unions of cells correspond to images of manifolds whose fundamental classes in locally finite homology are dual to the Thom classes, as in Definition 4.6 of [15], and thus are appropriate for elementary intersection theory. We can generalize the Fox-Neuwirth cell structure to a family of such in which specified sets of coordinates other than the first $a_{i}$ coordinates must be equal, with the resulting cohomology classes being independent of which are chosen. We then use that the cup product of Thom classes of two varieties is the Thom class of their intersection (when transversal). For example the set of configurations in which the first two points have their first $a_{1}$ coordinates agree intersected with the set in which the the first two points have the next $b_{1}$ coordinates equal will of course be the set in which the first two points have their first $a_{1}+b_{1}$ equal, and so forth, showing that the intersection product in the Fox-Neuwirth cell structure corresponds to the product structure of the associated Thom classes.

Using the intersection product, it is straightforward to compute the cup product structure appearing in Figure 4 on $H^{*}\left(B \mathcal{S}_{4} ; \mathbb{F}_{2}\right)$, using the skyline basis for convenience. The computations use observations that were made in the proof of Theorem 3.1 of cocycles which are coboundaries. Using the graphical skyline presentation, multiplication consists simply of "stacking columns in all possible ways", with a result of zero if a vertical line does not continue for the entire height of the column. See the end of Section 5 for the general recipe, and Section 6 of 15 , for a detailed account.

For a ring presentation, denote cohomology classes using only cochains, and let $\mathbf{x}=\operatorname{Symm}[1,0,0]$, $\mathbf{y}=[1,0,1]$ and $\mathbf{z}=[1,1,1]$. Then $H^{*}\left(B S_{4} ; \mathbb{F}_{2}\right) \cong \mathbb{F}_{2}[\mathbf{x}, \mathbf{y}, \mathbf{z}] /(\mathbf{x z})$. This presentation is deceptively simple. At present there are only recursive presentations of the relations in the cohomology rings of general symmetric groups. 

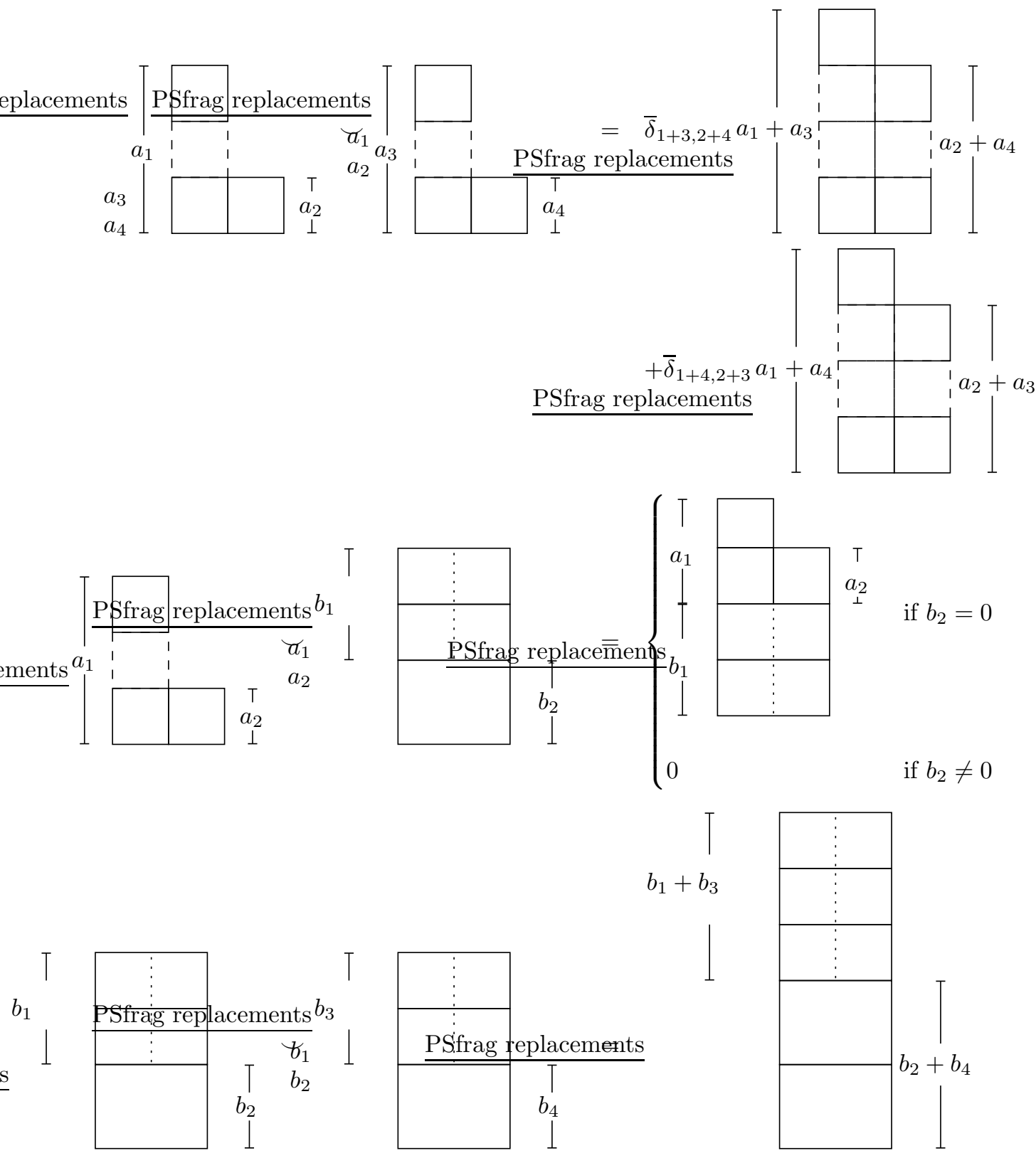

FIgURE 4. The cup product structure on $H\left(B \mathcal{S}_{4}\right)$. Here, $\bar{\delta}_{i+j, k+\ell}$ is $1+\delta\left(a_{i}+a_{j}, a_{k}+a_{\ell}\right) \in$ $\mathbb{F}_{2}$ where $\delta$ is the usual Kronecker delta function.

4.2. Hopf ring structure. We have found that the best way to organize the relations in the cohomology rings of symmetric groups, which seem inherently recursive, is through a Hopf ring structure

Definition 4.4. A Hopf ring is a ring object in the category of coalgebras. Explicitly, a Hopf ring is vector space $V$ with two multiplications, one comultiplication, and an antipode $(\odot, \cdot, \Delta, S)$ such that the 
first multiplication forms a Hopf algebra with the comultiplication and antipode, the second multiplication forms a bialgebra with the comultiplication, and these structures satisfy the distributivity relation

$$
\alpha \cdot(\beta \odot \gamma)=\sum_{\Delta \alpha=\sum a^{\prime} \otimes a^{\prime \prime}}\left(a^{\prime} \cdot \beta\right) \odot\left(a^{\prime \prime} \cdot \gamma\right) .
$$

Hopf rings were introduced by Milgram 21, and arise in topology as one structure governing the homology of infinite loop spaces which represent ring spectra. We give examples arising from algebra in Section 2 of [15] and will give some explicit calculations below.

Definition 4.5. Let $\mathbf{a}=\left[a_{1}, \ldots, a_{n-1}\right]$, and by convention set $a_{0}=a_{n}=0$. The coproduct of $\mathbf{a} \in \mathrm{FN}_{n}{ }^{*}$, is the sum $\Delta \mathbf{a}=\sum_{a_{i}=0}\left[a_{1}, \ldots, a_{i-1}\right] \otimes\left[a_{i+1}, \ldots, a_{n}\right]$ in $\bigoplus_{i+j=n} \mathrm{FN}_{i}{ }^{*} \otimes \mathrm{FN}_{j}{ }^{*}$.

Thus if $a_{i}>0$ for $1 \leq i \leq n-1$ then a will be primitive.

Definition 4.6. The transfer product of $\mathbf{a} \in \mathrm{FN}_{i}{ }^{*}$ and $\mathbf{b} \in \mathrm{FN}_{j}{ }^{*}$, denoted $\mathbf{a} \odot \mathbf{b}$, is the sum of cochains in $\mathrm{FN}_{i+j}{ }^{*}$ whose zero blocks are shuffles of the zero blocks of $\mathbf{a}$ and $\mathbf{b}$.

Theorem 4.7. The coproduct and transfer product induce a well-defined coproduct and product respectively on cohomology of $\bigoplus_{n} \mathrm{FN}_{n}{ }^{*}$.

Through the isomorphism of Theorem [2.9, the induced coproduct and product on cohomology agree with the direct sum over $i+j=n$ of natural maps and transfers respectively associated to the standard inclusions of symmetric groups $\mathcal{S}_{i} \times \mathcal{S}_{j} \hookrightarrow \mathcal{S}_{n}$.

Thus, the coproduct and transfer product on cohomology agree with the standard coproduct and the transfer product of Strickland and Turner [25. They show that the cohomology of the disjoint union of the symmetric groups is a Hopf ring with these along with the cup product, so we have the following corollary, once we define the intersection product between cochains in different summands of $\bigoplus_{n} \mathrm{FN}_{n}{ }^{*}$ to be zero.

Corollary 4.8. Through the isomorphism of Theorem [2.9, the intersection product, transfer product, and coproduct induce a Hopf semiring structure on the cohomology of $\bigoplus_{n} \mathrm{FN}_{n}{ }^{*}$. With mod two coefficients, the identity map defines an antipode which with these structures yields a Hopf ring.

An algebraic proof of this corollary is also possible. At the chain level, $(\cdot, \Delta)$ and $(\odot, \Delta)$ both induce bialgebra structures on $\bigoplus_{n} \mathrm{FN}_{n}{ }^{*}$, but the Hopf ring distributivity relation fails. However, there is a Hopf semiring structure on the subcomplex of symmetrized chains. This is analogous to rings of symmetric invariants, which are treated in Section 2 of [15]. For any algebra $A$ which is flat over its ground ring $R$, the total symmetric invariants, $\bigoplus_{n}\left(A^{\otimes n}\right)^{\mathcal{S}_{n}}$, forms a Hopf semiring. The bialgebra structures hold before taking invariants, but Hopf ring distributivity requires taking invariants.

We can use Hopf ring distributivity of the cup product over the transfer product to understand the cup product. Recall that $\operatorname{Symm}(\mathbf{a})$ denote the sum of all cochains related to a by a block-permutation. To compute $\operatorname{Symm}([3,0,2,2,2]) \cdot[\operatorname{Symm}([4,0,2,0,2])]$, we can decompose $\operatorname{Symm}[4,0,2,0,2]$ as $[4] \odot[2,0,2]$. Now, $\Delta(\operatorname{Symm}([3,0,2,2,2]))=[3] \otimes[2,2,2]$ plus terms which must cup to zero with the chains in our decomposition of Symm $[4,0,2,0,2])$ because they lie in the cohomology of other symmetric groups. Thus we have

$$
\begin{aligned}
\operatorname{Symm}([3,0,2,2,2]) \cdot \operatorname{Symm}([4,0,2,0,2]) & =(([3] \cdot[4]) \odot([2,2,2] \cdot[2,0,2])) \\
& =[7] \odot[4,2,4] \\
& =[7,0,4,2,4]+[4,2,4,0,7] \\
& =\operatorname{Symm}([7,0,4,2,4]) .
\end{aligned}
$$


4.3. Hopf ring presentation. We now develop the most basic classes, whose cup and transfer products will yield all mod-two cohomology of symmetric groups.

Definition 4.9. Let $\mathbf{g}_{\ell, n}$ be the basis element of $\left(\mathrm{FN}_{n 2^{\ell}}\right)^{n\left(2^{\ell}-1\right)}$ with $n$ 0-blocks, each of which consists of $\left(2^{\ell}-1\right)$ consecutive entries of 1 . Let $\gamma_{\ell, n}=\left[\mathbf{g}_{\ell, n}\right]$.

For example, $\mathbf{g}_{2,3}=[1,1,1,0,1,1,1,0,1,1,1] \in C^{9}\left(B \mathcal{S}_{12}\right)$. The following is an elaboration of the main result our paper with Salvatore [15, with Fox-Neuwirth cochain representatives now given.

Theorem 4.10. As a Hopf ring, $H^{*}\left(\coprod_{n} B \mathcal{S}_{n} ; \mathbb{F}_{2}\right)$ is generated by the classes $\gamma_{\ell, n} \in H^{n\left(2^{\ell}-1\right)}\left(B \mathcal{S}_{n 2^{\ell}}\right)$, along with unit classes on each component. The coproduct of $\gamma_{\ell, n}$ is given by

$$
\Delta \gamma_{\ell, n}=\sum_{i+j=n} \gamma_{\ell, i} \otimes \gamma_{\ell, j} .
$$

Relations between transfer products of these generators are given by

$$
\gamma_{\ell, n} \odot \gamma_{\ell, m}=\left(\begin{array}{c}
n+m \\
n
\end{array}\right) \gamma_{\ell, n+m} .
$$

The antipode is the identity map. Cup products of generators on different components are zero, and there are no other relations between cup products of generators.

For example, the cocycle $\operatorname{Symm}([4,3,4,1,4,3,4,0,3,3,3,0,1,0,1,0,1,0,0])$ is represented as the Hopf ring monomial $\gamma_{3,1} \gamma_{2,2}{ }^{2} \gamma_{1,4} \odot \gamma_{2,1}{ }^{3} \odot \gamma_{1,2} \odot \gamma_{1,1} \odot 1_{2}$ and, as we will see in the next section, the skyline diagram

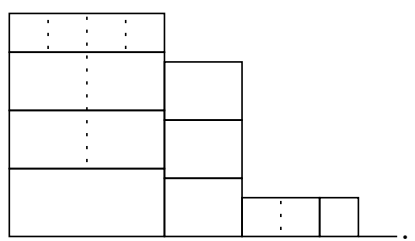

An immediate corollary of this theorem is that all cohomology classes have block-symmetric representatives, which was also found by Vassiliev [30].

\section{THE SKYLINE BASIS}

A Hopf ring monomial in classes $x_{i}$ is one of the form $f_{1} \odot f_{2} \odot \cdots \odot f_{k}$, where each $f_{j}$ is a monomial under the product in the $x_{i}$. Because of Hopf ring distributivity, it is convenient to use Hopf ring monomials in chosen generators to span a Hopf ring. In this section, we give a graphical presentation of a Hopf ring monomial basis for the mod-two cohomology of symmetric groups, using both spatial dimensions to represent the two products.

Definition 5.1. A gathered monomial in the cohomology of symmetric groups is a Hopf ring monomial in the generators $\gamma_{\ell, n}$ where such $n$ are maximal or equivalently the number of transfer products which appear is minimal.

For example, $\gamma_{1,4} \gamma_{2,2}{ }^{3} \odot \gamma_{1,2} \gamma_{2,1}{ }^{3}=\gamma_{1,6} \gamma_{2,3}{ }^{3}$. Gathered monomials such as the latter in which no transfer products appear are building blocks for general gathered monomials.

Definition 5.2. A gathered block is a monomial of the form $\prod_{i} \gamma_{\ell_{i}, n_{i}}{ }^{d_{i}}$, where the product is the cup product. Its profile is defined to be the collection of pairs $\left(\ell_{i}, d_{i}\right)$.

Non-trivial gathered blocks must have all of the numbers $2^{\ell_{i}} n_{i}$ equal, and we call this number divided by two the width. We assume that the factors are ordered from smallest to largest $n_{i}$ (or largest to smallest $\left.\ell_{i}\right)$, and then note that $n_{i}=2^{\ell_{1}-\ell_{i}} n_{1}$. 
Proposition 5.3. A gathered monomial can be written uniquely as the transfer product of gathered blocks with distinct profiles. Gathered monomials form a canonical additive basis for the cohomology of $\coprod_{n} B \mathcal{S}_{n}$.

Graphically, we represent $\gamma_{\ell, n}$ by a rectangle of width $n \cdot 2^{\ell}$ and height $1-\frac{1}{2^{\ell}}$, so that its area corresponds to its degree. We represent $1_{n}$ by an edge of width $n$ (a height-zero rectangle). A gathered block, which is a product of $\gamma_{\ell, n}$ for fixed $n \cdot 2^{\ell}$, is represented by a single column of such rectangles, stacked on top of each other, with order which does not matter. A gathered monomial is represented by placing such columns next to each other, which we call the skyline diagram of the monomial. We also refer to the gathered monomial basis as the skyline basis to emphasize this presentation.

In terms of skyline diagrams, the coproduct can be understood by introducing vertical dashed lines in the rectangles representing $\gamma_{\ell, n}$, dividing the rectangle into $n$ equal pieces. The coproduct is then given by dividing along all existing columns and vertical dashed lines of full height and then partitioning them into two to make two new skyline diagrams.

The transfer product corresponds to placing two column Skyline diagrams next to each other and merging columns with the same constituent blocks, with a coefficient of zero if any of those column widths share a one in their dyadic expansion.

For cup product, we start with two column diagrams and consider all possible ways to split each into columns, along either original boundaries of columns or along the vertical lines of full height internal to the rectangles representing $\gamma_{\ell, n}$. We then match columns of each in all possible ways up to automorphism, and stack the resulting matched columns to get a new set of columns, as we saw for $B \mathcal{S}_{4}$.

See Figure 5 for illustrations of cup product, which thus also involve the coproduct and transfer product. See Section 6 of [15] for thorough treatments of all of these structures.

We can use our formula for multiplication to see for example that Vassiliev's conjecture that $d$ th powers in the cohomology of $\overline{\operatorname{Conf}}_{n}\left(\mathbb{R}^{d}\right)$ are zero is not true. The cohomology of $\overline{\operatorname{Conf}}_{n}\left(\mathbb{R}^{d}\right)$ is the quotient of that of $\overline{\operatorname{Conf}}_{n}\left(\mathbb{R}^{\infty}\right)$ setting skyline diagrams with some block height greater than or equal to $d$ to zero. Then

for example in $\overline{\operatorname{Conf}}_{6}\left(\mathbb{R}^{3}\right)$ the cube of

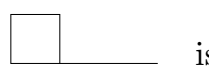

is

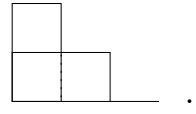

\section{The cohomology of $B \mathcal{S}_{\infty}$ as an algebra over the Steenrod algebra}

6.1. Nakaoka's theorem revisited. The infinite symmetric group plays a special role in algebraic topology. Let $\Omega^{\infty} S^{\infty}$ denote the direct limit, under suspension of maps, of the space of based maps from $S^{d}$ to itself. The Barratt-Priddy-Quillen-Segal theorem [4] says that the cohomology of $\Omega^{\infty} S^{\infty}$ is isomorphic to that of $B \mathcal{S}_{\infty}$.

The map $B \mathcal{S}_{n} \rightarrow B \mathcal{S}_{n+1}$ induced by inclusion induces the map on cohomology which sends a skyline diagram with at least one empty column to that obtained by removing that column, and is zero on diagrams with no empty columns. The inverse limit is thus spanned by skyline diagrams with a finite number of non-empty columns, along with infinitely many empty columns, which we ignore. We let the width of such a diagram be the total width of the non-empty columns. Multiplication is through essentially the same algorithm as in for $B \mathcal{S}_{n}$, which generally increases width unless for example some diagram is raised to a power of two.

Definition 6.1. A column is even if every block type occurs an even number of times, and odd if at least one block type occurs an odd number of times. Define the two-root of a skyline diagram $D$ consisting of a single column as the odd column $R$ such that $R^{2^{p}}=D$.

Theorem 6.2 (after Nakaoka 22]). The mod-two cohomology of $B \mathcal{S}_{\infty}$ is polynomial, generated by diagrams consisting of a single odd column. 


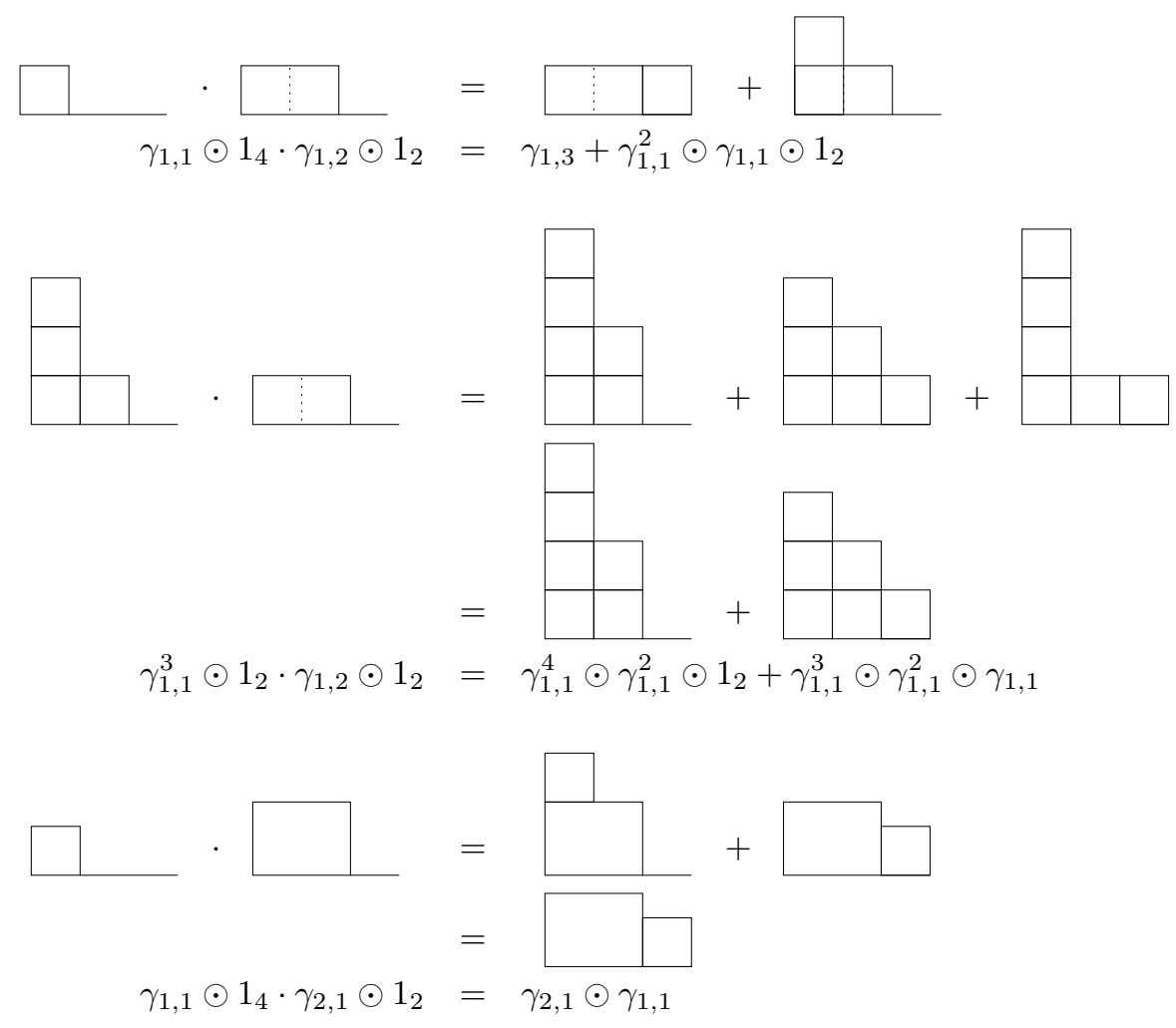

FiguRE 5. Examples of product computations in $H^{*}\left(B \mathcal{S}_{6} ; \mathbb{Z} / 2\right)$ expressed in skyline and Hopf monomial notation

This theorem along with Theorem 4.10 extends Nakaoka's theorem by giving cochain representatives in $\mathrm{FN}_{\infty}{ }^{*}$ for generators. This result should also be compared with the explicit calculation of homology primitives by Wellington 31 .

Proof. We filter the cohomology of $B \mathcal{S}_{\infty}$, as represented by skyline diagrams, by width. If $D$ is a diagram with columns $C_{1}, \ldots, C_{n}$, let $R_{1}, \ldots, R_{n}$ be their two-roots with $R_{i}{ }^{2^{p_{i}}}=C_{i}$. By abuse let $R_{i}$ denote the diagram which consists of $R_{i}$ as its only non-empty column. Using the algorithm to multiply skyline diagrams by stacking columns, $D=\prod_{i} R_{i}{ }^{2^{p_{i}}}$ modulo diagrams of lower filtration. Thus the associated graded to the width filtration is a polynomial algebra on diagrams consisting of a single odd column. So the cohomology of $B \mathcal{S}_{\infty}$ itself must be polynomial.

In the course of proof, we see that the change of basis between the skyline basis and the monomial basis arising from the theorem is non-trivial but straightforward.

6.2. Steenrod structure. Next we focus on the Steenrod algebra structure on the cohomology of $B \mathcal{S}_{\infty}$ or equivalently $\Omega^{\infty} S^{\infty}$. To do so it is best to use the connection between the cohomology of groups and invariant theory which has been a fundamental tool in the subject. Recall for example from Chapters 3 and 4 of [1] that if $H$ is a subgroup of $G$ then the Weyl group of $H$ in $G$ acts on the cohomology of $H$. Moreover, the restriction map from the cohomology of $G$ to that of $H$ has image in the invariants under this action, namely $\left(H^{*}(B H)\right)^{W(H)}$. 
In the study of the cohomology of symmetric groups, the invariant theory which arises is classical. Let the subgroup $H$ in question be the subgroup $(\mathbb{Z} / 2)^{n} \subset \mathcal{S}_{2^{n}}$ defined by having $(\mathbb{Z} / 2)^{n}$ act on itself, which we call $V_{n}$. The cohomology of $V_{n}$ is that of $\prod_{n} \mathbb{R} P^{\infty}$, namely $\mathbb{F}_{2}\left[x_{1}, \ldots, x_{n}\right]$. If we view the action of $V_{n}$ on itself as given by linear translations on the $\mathbb{F}_{2}$-vector space $\oplus_{n} \mathbb{F}_{2}$, then we can see that the normalizer of this subgroup is isomorphic to all affine transformations of $\left(\mathbb{F}_{2}\right)^{n}$. The Weyl group is thus $G L_{n}\left(\mathbb{F}_{2}\right)$. Moreover, the action is by linear action on the variables $x_{1}, \ldots, x_{n}$.

The invariants $\mathbb{F}_{2}\left[x_{1}, \ldots, x_{n}\right]^{G L_{n}\left(\mathbb{F}_{2}\right)}$, studied a century ago, are known as Dickson algebras. Because permutation matrices are in $G L_{n}\left(\mathbb{F}_{2}\right)$ the invariants are in particular symmetric polynomials. But for example there is never a $G L_{n}\left(\mathbb{F}_{2}\right)$ invariant in degree one, since the lone symmetric invariant $x_{1}+\cdots+x_{n}$ is not invariant under the linear substitution $x_{1} \mapsto x_{1}+x_{2}$ (and $x_{i} \mapsto x_{i}$ for $i>1$ ). Dickson's theorem is that as rings these invariants are polynomial algebras on generators $d_{k, \ell}$ in dimensions $2^{k}\left(2^{\ell}-1\right)$ where $k+\ell=n$. For example, $\mathbb{F}_{2}\left[x_{1}, x_{2}\right]^{G L_{2}\left(\mathbb{F}_{2}\right)}$ is generated by an invariant in degree two, namely $x_{1}^{2}+x_{2}^{2}+x_{1} x_{2}$, along with $x_{1}^{2} x_{2}+x_{1} x_{2}^{2}$ in degree three.

This connection to invariant theory allows us to determine the action of the Steenrod algebra. The standard starting point is that of the cohomology of $\mathbb{R} P^{\infty}$, which allows us to understand the Steenrod structure on that of $B V_{n} \simeq \prod_{n} \mathbb{R} P^{\infty}$ by the Cartan formula. Because the Steenrod action is defined by squaring individual variables, which is a linear operation over $\mathbb{F}_{2}$, the $G L_{n}$-invariants are preserved by the Steenrod action. For example

$$
S q^{1}\left(x_{1}{ }^{2}+x_{2}{ }^{2}+x_{1} x_{2}\right)=0 \cdot x_{1}^{3}+0 \cdot x_{2}{ }^{2}+x_{1}{ }^{2} x_{2}+x_{1} x_{2}{ }^{2} .
$$

In [16] Hu'ng calculated the Steenrod squares on Dickson classes as given by

$$
S q^{i} d_{k, \ell}= \begin{cases}d_{k^{\prime}, \ell^{\prime}} & i=2^{k}-2^{k^{\prime}} \\ d_{k^{\prime}, \ell^{\prime}} d_{k^{\prime \prime}, \ell^{\prime \prime}} & i=2^{n}+2^{k}-2^{k^{\prime}}-2^{k^{\prime \prime}}, \quad k^{\prime} \leq k<k^{\prime \prime} \\ d_{k, \ell} & i=2^{k}\left(2^{\ell}-1\right) \\ 0 & \text { otherwise. }\end{cases}
$$

Turning our attention back to symmetric groups, the transfer product in cohomology is induced by a stable map. Thus there is a Cartan formula for transfer product as well, and it suffices to understand Steenrod structure on Hopf ring generators. In [15] we prove the following.

Theorem 6.3. The restriction of $\gamma_{\ell, 2^{k}}$ with $k+\ell=n$ to the elementary abelian subgroup $V_{n}$ is the Dickson invariant $d_{k, \ell}$.

This theorem, Hu'ng's calculation above and the fact that the direct some of the restriction map to $V_{n}$ and the coproduct $\Delta$ is injective allow us to understand the Steenrod on the $\gamma_{\ell, 2^{k}}$.

Theorem 6.4. A Steenrod square on $\gamma_{\ell, 2^{k}}$ is represented in the skyline basis by the sum of all diagrams which are of full width, with at most two boxes stacked on top of each other, and with the width of columns delineated by any of the vertical lines (of full height) at least $\ell$.

See Figure 6 for some examples using the Cartan formula and this result.

6.3. Madsen's theorem revisited. One of the first questions to ask about an algebra $R$ over the Steenrod algebra is its vector space of indecomposables, which we denote $\operatorname{Indec}_{\mathcal{A}-\mathrm{Alg}} R$. If $R$ is the cohomology of a space, it is this vector space which can evaluate non-trivially on the Hurewicz homomorphism from homotopy to homology. In the case of the infinite symmetric group (and thus $\Omega^{\infty} S^{\infty}$ ), this question is reduced to one in invariant theory. This connection was first made by Madsen forty years ago [19], but there is still much to learn about the resulting algebraic question [17.

Our starting point is Theorem 6.2, which immediately determines the indecomposables of $H^{*}\left(B \mathcal{S}_{\infty}\right)$ as an algebra. 


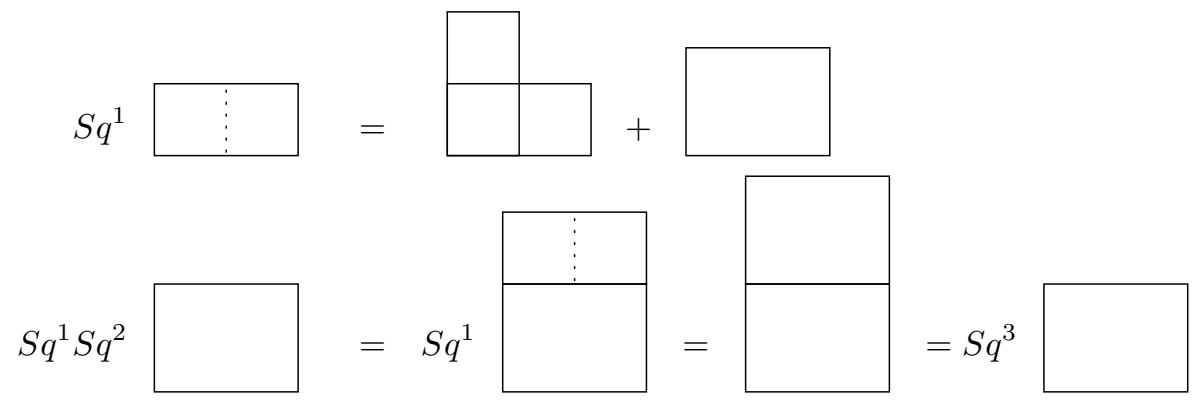

Figure 6. Steenrod algebra action on some elements of the skyline basis for $B \mathcal{S}_{4}$. The second calculation uses the first through the Cartan formula, with the term $\left(\gamma_{1,1}^{2} \odot \gamma_{1,1}\right) \cdot \gamma_{2,1}$ being zero.

Definition 6.5. Define the width splitting of $\operatorname{Indec}_{A l g} H^{*}\left(B \mathcal{S}_{\infty}\right)$, the algebra indecomposables of $H^{*}\left(B \mathcal{S}_{\infty}\right)$, by letting $W_{k}$ denote the span of indecomposables which are represented by single columns of width $2^{k-1}$. Define the width filtration through the increasing sums $\bigoplus_{k=1}^{n} W_{k}$.

We have the following remarkable connection between topology and invariant theory, which goes back at least to work of Selick (and Cohen and Peterson) [24.

Theorem 6.6. The associated graded of the width filtration on $\operatorname{Indec}_{A l g} H^{*}\left(B \mathcal{S}_{\infty}\right)$ is isomorphic as $\mathcal{A}$ modules to the direct sum of Dickson algebras $\bigoplus_{n} \mathbb{F}_{2}\left[x_{1}, \ldots, x_{n}\right]^{G L_{n}\left(\mathbb{F}_{2}\right)}$.

Proof. In the description for Steenrod action on the Hopf ring generators $\gamma_{\ell, 2^{k}}$ of Theorem 6.4 there is exactly one term in which there is just one column. This term corresponds to the formula for squares on Dickson generators as in Equation 1 (by replacing $d_{k, \ell}$ by $\gamma_{\ell, 2^{k}}$ ). All other terms have more than one column, and thus modulo decomposables are lower in the width filtration.

The fact that for an algebra $R$ over the Steenrod algebra, the quotient map induces an isomorphism of graded vector spaces $\operatorname{Indec}_{\mathcal{A}-\mathrm{Alg}} R \cong \operatorname{Indec}_{\mathcal{A}-\text { Mod }}\left(\operatorname{Indec}_{\mathrm{Alg}} R\right)$ implies that $\operatorname{Indec}_{\mathcal{A}-\operatorname{Alg}} H^{*}\left(\Omega^{\infty} S^{\infty}\right)$ is a quotient of $\bigoplus_{n} \operatorname{Indec}_{\mathcal{A}-\mathrm{Mod}} \mathbb{F}_{2}\left[x_{1}, \ldots, x_{n}\right]^{G L_{n}\left(\mathbb{F}_{2}\right)}$. To date, the most extensive calculations have been of the dual primitives in homology by Wellington [31 and recently by Zare [32. The Steenrod structure on the algebra indecomposables was put to great use by Campbell, Cohen, Peterson and Selick 10, 9 . The skyline basis in cohomology gives a distinct approach, which may be especially useful in tandem with homology.

\section{REFERENCES}

1. Alejandro Adem and R. James Milgram, Cohomology of finite groups, Grundlehren der Mathematischen Wissenschaften [Fundamental Principles of Mathematical Sciences], vol. 309, Springer-Verlag, Berlin, 1994. MR MR1317096 (96f:20082)

2. M. F. Atiyah, Thom complexes, Proc. London Math. Soc. (3) 11 (1961), 291-310. MR 0131880 (24 \#A1727)

3. D. Ayala and R. Hepworth, Configurations spaces and theta-n, arXiv:1202.2806.

4. Michael Barratt and Stewart Priddy, On the homology of non-connected monoids and their associated groups, Comment. Math. Helv. 47 (1972), 1-14. MR MR0314940 (47 \#3489)

5. Clemens Berger, Opérades cellulaires et espaces de lacets itérés, Ann. Inst. Fourier (Grenoble) 46 (1996), no. 4, 11251157. MR 1415960 (98c:55011)

6. - Combinatorial models for real configuration spaces and $E_{n}$-operads, Operads: Proceedings of Renaissance Conferences (Hartford, CT/Luminy, 1995), Contemp. Math., vol. 202, Amer. Math. Soc., Providence, RI, 1997 , pp. 37-52. MR 1436916 (98j:18014)

7. Terrence P. Bisson and André Joyal, Q-rings and the homology of the symmetric groups, Operads: Proceedings of Renaissance Conferences (Hartford, CT/Luminy, 1995), Contemp. Math., vol. 202, Amer. Math. Soc., Providence, RI, 1997, pp. 235-286. MR MR1436923 (98e:55021) 
8. P. Blagojević and G. Ziegler, Convex equipartitions via equivariant obstruction theory, arXiv:1202.5504.

9. H. E. A. Campbell, F. R. Cohen, F. P. Peterson, and P. S. Selick, Self-maps of loop spaces. II, Trans. Amer. Math. Soc. 293 (1986), no. 1, 41-51. MR 814910 (87e:55010a)

10. H. E. A. Campbell, F. P. Peterson, and P. S. Selick, Self-maps of loop spaces. I, Trans. Amer. Math. Soc. 293 (1986), no. 1, 1-39. MR 814910 (87e:55010a)

11. Eugenia Cheng and Aaron Lauda, Higher-dimensional categories, an illustrated guidebook., available at http://www.cheng.staff.shef.ac.uk/.

12. Frederick R. Cohen, Thomas J. Lada, and J. Peter May, The homology of iterated loop spaces, Springer-Verlag, Berlin, 1976. MR MR0436146 (55 \#9096)

13. Mark Feshbach, The mod 2 cohomology rings of the symmetric groups and invariants, Topology 41 (2002), no. 1, 57-84. MR MR1871241 (2002h:20074)

14. R. Fox and L. Neuwirth, The braid groups, Math. Scand. 10 (1962), 119-126. MR MR0150755 (27 \#742)

15. Chad Giusti, Paolo Salvatore, and Dev P. Sinha, The mod 2 cohomology of symmetric groups as a hopf ring over the steenrod algebra, arXiv:0909.3292.

16. Nguyên H. V. Hung, The action of the Steenrod squares on the modular invariants of linear groups, Proc. Amer. Math. Soc. 113 (1991), no. 4, 1097-1104. MR 1064904 (92c:55018)

17. Nguyen H. V. Hu'ng and Franklin P. Peterson, Spherical classes and the Dickson algebra, Math. Proc. Cambridge Philos. Soc. 124 (1998), no. 2, 253-264. MR MR1631123 (99i:55021)

18. André Joyal, Duality and $\theta$-categories.

19. Ib Madsen, On the action of the Dyer-Lashof algebra in $H_{*}(G)$, Pacific J. Math. 60 (1975), no. 1, 235-275. MR 0388392 (52 \#9228)

20. R. James Milgram, Iterated loop spaces, Ann. of Math. (2) 84 (1966), 386-403. MR MR0206951 (34 \#6767)

21. _ The mod 2 spherical characteristic classes, Ann. of Math. (2) 92 (1970), 238-261. MR MR0263100 (41 \#7705)

22. Minoru Nakaoka, Homology of the infinite symmetric group, Ann. of Math. (2) 73 (1961), 229-257. MR MR0131874 (24 \#A1721)

23. M. Salvetti, Topology of the complement of real hyperplanes in $\mathbf{C}^{N}$, Invent. Math. 88 (1987), no. 3, 603-618. MR 884802 (88k:32038)

24. Paul Selick, On the indecomposability of certain sphere-related spaces, Current trends in algebraic topology, Part 1 (London, Ont., 1981), CMS Conf. Proc., vol. 2, Amer. Math. Soc., Providence, R.I., 1982, pp. 359-372. MR 686125 (84b:55012)

25. Neil P. Strickland and Paul R. Turner, Rational Morava E-theory and DS ${ }^{0}$, Topology 36 (1997), no. 1, $137-151$. MR MR1410468 (97g:55005)

26. Dai Tamaki, Cellular stratified spaces I : Face categories and classifying spaces., arXiv:1106.3772.

27. Cellular stratified spaces II: Basic constructions., arXiv:1111.4774.

28. _ The Salvetti complex and the little cubes., math/0602085.

29. — A dual Rothenberg-Steenrod spectral sequence, Topology 33 (1994), no. 4, 631-662. MR 1293304 (95f:55019)

30. V. A. Vassiliev, Complements of discriminants of smooth maps: topology and applications, Translations of Mathematical Monographs, vol. 98, American Mathematical Society, Providence, RI, 1992, Translated from the Russian by B. Goldfarb. MR MR1168473 (94i:57020)

31. Robert J. Wellington, The unstable Adams spectral sequence for free iterated loop spaces, Mem. Amer. Math. Soc. 36 (1982), no. 258, viii+225. MR 646741 (83c:55028)

32. Hadi Zare, On spherical classes in $H_{*}(Q X)$, arXiv:1101.1215.

Mathematics Department, Willamette University

E-mail address: cgiusti@willamette.edu

Mathematics Department, University of Oregon

E-mail address: dps@math.uoregon.edu 